\title{
Expression of human mammaglobin as a marker of bone marrow micrometastasis in breast cancer
}

\author{
YUNJIANG LIU, LI MA, XIANYI LIU and LI WANG \\ Department of Breast Center, No. 4 Hospital of Hebei Medical University, Shijiazhuang 050011, P.R. China
}

Received September 21, 2011; Accepted December 19, 2011

DOI: $10.3892 / \mathrm{etm} .2011 .429$

\begin{abstract}
The aim of the present study was to detect the expression of human mammaglobin (hMAM) mRNA in the bone marrow $(\mathrm{BM})$ of patients with breast cancer and determine the relationship between micrometastasis and clinicopathological parameters as well as selected molecular markers and breast cancer prognosis. The expression of hMAM mRNA in the BM of patients with breast cancer was determined by RT-PCR. The expression of ER, PR and Cath-D in cancer tissues was detected by immunohistochemistry. A positive expression rate for hMAM of $38.2 \%$ in 102 patients with stage I-III breast cancer was found. The expression of hMAM was higher in patients with $\mathrm{T}_{2-3}(>2 \mathrm{~cm})$ tumors than in those with $\mathrm{T}_{1}$ tumors $(\leq 2 \mathrm{~cm})\left(\chi^{2}=19.20, \mathrm{P}=0.001\right)$ and in patients with stage II or III tumors than in patients with stage I tumors $\left(\chi^{2}=15.101, \mathrm{P}=0.001\right)$. The expression of hMAM in the BM of breast cancer patients categorized as grade 1 was lower than that in those of grade 2 or $3\left(\chi^{2}=8.522, \mathrm{P}=0.014\right)$, and hMAM expression was related to the pathological type of tumor $\left(\chi^{2}=6.892, \mathrm{P}=0.032\right)$ and the degree of axillary lymph node metastasis $\left(\chi^{2}=14.050, \mathrm{P}=0.001\right)$. The expression of hMAM in $\mathrm{BM}$ was much higher in patients with $\mathrm{ER}(-)$ or ER(+) tumors than in those with $\mathrm{ER}(++$ or +++$)\left(\chi^{2}=11.800, \mathrm{P}=0.003\right)$, and those with PR $\left(\chi^{2}=8.759, \mathrm{P}=0.013\right)$. hMAM expression in $\mathrm{BM}$ was also significantly positively correlated with Cath-D expression $\left(\chi^{2}=6.623, \mathrm{P}=0.036\right)$. However, no correlation was found between hMAM expression and patient age $\left(\chi^{2}=1.056\right.$, $\mathrm{P}=0.304)$. There was a strong correlation between patients with positive expression of hMAM in the BM and the presence of distant metastases $(\mathrm{P}=0.009)$. In conclusion, micrometastasis in the BM correlates with certain clinical pathological parameters and several tumor markers. Patients with positive expression of
\end{abstract}

Correspondence to: Dr Yunjiang Liu, Department of Breast Center, The Fourth Hospital of Hebei Medical University, Shijiazhuang 050011, P.R. China

E-mail: 1yj818326@126.com

Key words: breast cancer, bone marrow micrometastasis, human mammaglobin, biological factor, RT-PCR, immunohistochemistry, prognosis
hMAM in the BM have a greater chance of distant metastasis and poor prognosis. The detection of micrometastasis may be one of the most advantageous markers for predicting the prognosis of breast cancer.

\section{Introduction}

Breast cancer is the most common malignant disease in the world affecting women. In these patients, it is not the primary tumor but its metastases at distant sites that are the main cause of death. Clinical trials suggest that most cases of breast cancer may be systemic, and in approximately $25-30 \%$ of breast cancer patients in whom axillary lymph nodes test negative, distant metastases appear within 5 years after surgery, sometimes resulting in death (1). Systemic adjuvant therapy can help eradicate breast cancer cells that may have already spread to distant sites by the time of diagnosis and could remain avascular for a long period of time but retain the potential to multiply and induce metastasis if they became active (2). Human mammaglobin (hMAM) is expressed at a lower level in normal breast epithelium, but at a higher level in breast cancer tissue, and is not expressed in normal blood and bone marrow (BM). We detected the expression of hMAM mRNA in the BM of patients with breast cancer, and analyzed the relationships between micrometastasis in the BM and clinicopathological parameters as well as the prognosis of breast cancer. In addition, using this method, we were able to make a judgment regarding the likelihood of breast cancer BM micrometastasis and discuss its relationships with ER, PR, Cath-D and other markers. Our purpose was to study the regulation of breast cancer metastasis, in order to provide markers to predict prognosis and provide the foundation for individualized treatment.

\section{Materials and methods}

Patients. Of the breast cancer patients treated in our hospital from 2002 to 2003, 102 cases were chosen stochastically. All were female, diagnosed by pathology, and were aged from 30 to 76 years (49.2 average). Twelve patients were at stage I, 59 at stage II, and 31 at stage III. All patients were treated surgically, without radiotherapy or chemotherapy prior to surgery. Fifty-three cases were infiltrating ductal carcinoma (IDC), 35 were infiltrating lobular carcinoma (ILC), and 14 were other categories. Ten cases of benign breast disease were used as the 
control group, and these included hyperplasia of the mammary glands and fibroadenoma. Ten milliliters of bone marrow was obtained preoperatively by bone marrow aspiration at the anterior superior iliac spine, with informed consent, and stored at $-70^{\circ} \mathrm{C}$ until used for detection.

All patients were followed up from 13 to 60 months after surgery, 47 months on average. At the final follow-up, 8 cases had been diagnosed with distant metastases or had died, while all others survived disease-free.

RT-PCR, primers and amplification product evaluation. The hMAM primer was purchased from Shanghai-Sheng-Gong Bioengineering Co. The upstream primer was -AGC ACT GCT ACG CAG GCT CT-3' and the downstream primer, 5'-ATA AGA AAG AGA AGG TGT GG-3'; amplification product length $329 \mathrm{bp}$. The inner-reference $\beta$-actin upstream primer was 5'-ATC TGG CAC CAC ACC TTC TAC AAT GAG CTG CG-3' and the downstream primer, 5'-CGT CAT ACT CCT GCT TGC TGA TCC ACA TCT GC-3'; amplification product length $838 \mathrm{bp}$ (Fig. 1).

TRIzol was used to lyse the cells, and the guanidinium thiocyanate-phenol-chloroform one-step extraction method was used to obtain total-RNA. The RNA extracted was electrophoresed on a $1.5 \%$ agarose gel; the width and brightness of the 28S RNA band was double the $18 \mathrm{~S}$ band as determined under an ultraviolet transilluminator, which indicated good integrity of the extracted RNA.

The reaction parameters were as follow: $37^{\circ} \mathrm{C}$ reverse transcription for $50 \mathrm{~min}$ to obtain cDNA for PCR amplification, followed by cycling conditions of: $95^{\circ} \mathrm{C}$ denaturation, $5 \mathrm{~min}$, $94^{\circ} \mathrm{C}$, melting, $45 \mathrm{sec}, 56^{\circ} \mathrm{C}$ annealing, $1 \mathrm{sec}, 72^{\circ} \mathrm{C}$ extension, 1 sec, 35 cycles, with a final 5 min extension; $\beta$-actin and hMAM were amplified in the same tube.

We reached a positive diagnosis of BM metastasis when a clear and specific 329-bp hMAM band and an 838-bp $\beta$-actin band was found simultaneously. When the internal standard $\beta$-actin was positive but hMAM was negative, the case was diagnosed as BM metastasis-negative (Fig. 1).

Immunohistochemical technique and evaluation of the results. Streptavidin-peroxidase (S-P) immunohistochemistry, using a kit from Beijing Zhongshan Biotechnology Company, was performed using primary antibodies at the following dilutions: ER 1:240; PR 1:400; Cath-D 1:80. Both negative and positive controls were run alongside the samples; for the negative control PBS was used in place of the antibody, while for the positive control, directions supplied in the kit were followed.

Twenty high-power fields were selected using a light microscope, and at least 2,000 cells were counted, and then aggregated to analyze the positive cell population and staining intensity of the entire slice, which was judged according to the positive intensity as specified in the different kits. Levels of staining are indicated by (-) to represent negative staining, while positive staining was classified as $(+),(++)$ or $(+++)$.

Statistical analysis. Results were analyzed using SPSS 13.0 software, by the RxC Chi-square test and the quadruple tabular form exact probability test. Statistical significance was defined as $\mathrm{P}<0.05$

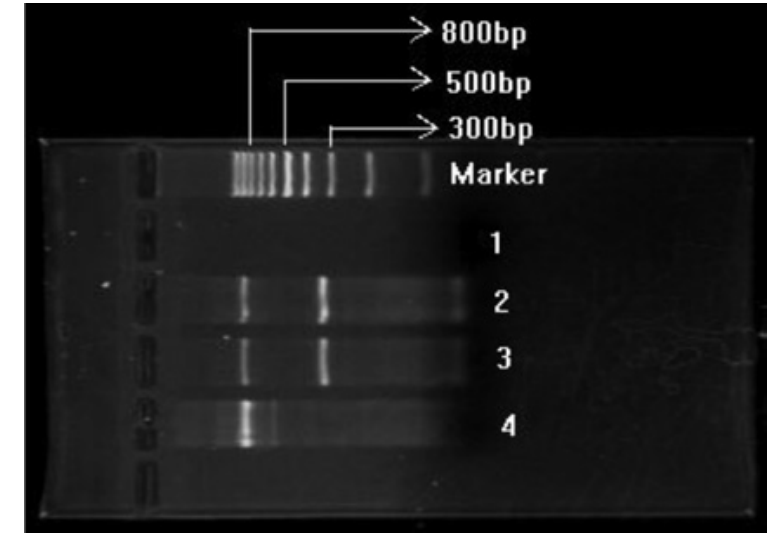

Figure 1. Expression of hMAM mRNA in the BM from patients with breast cancer. Marker, DNA ladder (100-1000 bp). Lane 1, negative control; lane 2, positive control; lane 3, positive expression of hMAM mRNA in BM; lane 4, negative expression of hMAM mRNA in BM.

\section{Results}

hMAM $m R N A$ expression in the BM of breast cancer patients. No mRNA expression of hMAM was found in the BM of 10 patients with benign breast disease. In contrast, positive expression of hMAM was found in the BM of 39 of 102 breast cancer patients; a positivity rate of $38.2 \%$. Statistical analysis revealed a significance level of $\mathrm{P}=0.014$.

Relationship between hMAM mRNA expression in the BM and size of the tumor. The expression of hMAM in the BM was greatly increased with increasing tumor size. The expression rate in patients with a tumor of size $<2,2-5$ and $>5 \mathrm{~cm}$ was $12.5 \%$ (2/16), 33.3\% (23/69) and 76.5\% (14/17), respectively; comparison among the three groups achieved a statistical significance $\left(\chi^{2}=19.20, \mathrm{P}=0.001\right)$ (Table I).

Relationship between hMAM mRNA expression in the BM and clinical stage. The rate of hMAM expression increased with advancing clinical stage; the expression rate for stage I was $8.3 \%$ (1/12), stage II, 30.5\% (18/59) and stage III, 64.5\% (20/31) $\left(\chi^{2}=15.101, \mathrm{P}=0.001\right)$ (Table I).

Relationship between hMAM mRNA expression in the BM and axillary lymph node metastasis. The expression rate of hMAM in the BM was higher in the group positive for axillary lymph node metastasis $(52.5 \%$; $27 / 51)$ than in the negative group $(23.5 \%$; 12/51); the result achieved statistical significance $\left(\chi^{2}=14.050, \mathrm{P}=0.001\right)$ (Table I). The positive group was further separated into two subgroups: A, those with 3 or fewer involved lymph nodes; and B, the remaining patients, with 4 or more. The results of ambi-comparisons showed that the rate of micrometastasis in the BM was higher in group B $(\geq 4)$ than in the negative group or group $\mathrm{A}(\leq 3)$, this result was also statistically significant $\left(\chi^{2}=4.464,14.060\right.$ respectively; $\mathrm{P}=0.035,0.001$ respectively).

Relationship between hMAM mRNA expression in BM and pathology. hMAM mRNA expression was much higher in the group with higher potential malignancy. The rate in patients with infiltrating ductal carcinoma was $45.3 \%$ (24/53), and the 
Table I. Relationship between expression of hMAM in the bone marrow of breast cancer patients and tumor size, metastasis to the lymph nodes, clinical stage, patient age, pathological types, histological grading and expression of ER, PR and Cath-D.

hMAM mRNA expression

\begin{tabular}{lllllll} 
& $\mathrm{n}$ & + & & Positive rate $(\%)$ & $\chi^{2}$-value \\
\hline
\end{tabular}

\section{Tumor size}

$<2 \mathrm{~cm}$

$2-5 \mathrm{~cm}$

$>5 \mathrm{~cm}$

16

69

17

14

46

2

23

12.5

33.3

14

82.4

Lymphatic metastasis

0
$1-3$
$\geq 4$

Clinical stage

I

II 59

51

39

12

26

25

16

10

17

23.5

14.050

61.5

68.0

$\begin{array}{lll}8 & 17 & 68.0\end{array}$

12

III

59
31

11

1

8.3

15.101

0.001

18

20

30.5

64.5

Age (years)

$\leq 50$

61

41

40

21

23

Pathological type

Infiltrating ductal carc. 53

Infiltrating lobular carc. $\quad 35$

Others

Histological grading

1
2
3

$\begin{array}{lll}30 & 25 & 5\end{array}$

$\begin{array}{lll}44 & 24 & 20\end{array}$

28

14

20

14

ER

-

$+\quad 22$

$++,+++$

27

11

11

16

11

412

$53 \quad 41$

PR

-

$+\quad 26$

$++,+++$

31
26
45

\begin{abstract}
15
\end{abstract}

13

16

13

$35 \quad 10$

Cath-D

\begin{tabular}{lrrrrr}
- & 13 & 9 & 4 & 30.8 & 6.623 \\
+ & 26 & 21 & 5 & 19.2 & 0.036 \\
,+++++ & 63 & 33 & 30 & 47.6 & \\
\hline
\end{tabular}

34.4

1.056

43.9

45.3

40.0

7.1

16.7

45.5

50.0

59.3

50.0

51.6

8.759

0.013

50.0

22.2

8.522

0.014

11.80

0.003

22.6

6.892

0.032

0.304

50.0
10

8

19.2 rate in patients with infiltrating lobular carcinoma was $40.0 \%$ (14/35). In contrast, in cases with a lower potential malignancy, the rate of hMAM expression was $7.1 \%(1 / 14)$; this group consisted of 4 cases of typical medullary carcinoma, 4 cases of tubular adenocarcinoma, 2 cases of atypical hyperplasia with canceration, 2 cases of mucinous adenocarcinoma, and 2 cases of Paget's disease. The rate of expression in infiltrating ductal carcinoma was close to that in infiltrating lobular carcinoma $\left(\chi^{2}=0.240, P=0.624\right)$. However, the difference between these two diseases and the group of low potential malignant diseases was statistically significant $\left(\chi^{2}=6.892, \mathrm{P}=0.032\right)$ (Table I).

Relationship between hMAM $m R N A$ expression in the BM and histological differentiation. The rate of hMAM mRNA expression in the BM increased with increasing histological grade. The rate of positive expression in grade 1 was $16.7 \%$ $(5 / 30)$, in grade $2,45.5 \%(20 / 44)$, and in grade $3,50.0 \%(14 / 28)$ $\left(\chi^{2}=8.522, \mathrm{P}=0.014\right)$ (Table I). 
Relationship between BM micrometastasis and expression of $E R$ and $P R$. The BM micrometastasis rate in the ER(-) group was $59.3 \%(16 / 27)$ and the rate in the $\mathrm{ER}(+)$ group was $50.0 \%$ $(11 / 22)$, while in the $\mathrm{ER}(++,+++)$ group it was $22.6 \%(12 / 53)$, $\left(\chi^{2}=11.800, \mathrm{P}=0.003\right)$. Likewise, the $\mathrm{BM}$ micrometastasis rate in the PR(-) group was $51.6 \%(16 / 31)$ and in the PR(+) group it was $50.0 \%(13 / 26)$, while in the $\mathrm{PR}(++,+++)$ group the rate was $22.2 \%(10 / 45)\left(\chi^{2}=8.759, \mathrm{P}=0.013\right)$ (Table I).

Relationship between BM micrometastasis and expression of Cath-D in breast cancer tissue. The expression rate of hMAM mRNA in the BM of the 102 patients with breast cancer increased concomitantly with the expression of Cath-D in the breast cancer tissues. The BM micrometastasis rate of the Cath-D(-) group was 30.8\% (4/13); and the BM micrometastasis rate in the Cath- $\mathrm{D}(+)$ group was $19.2 \%(5 / 26)$, and this rate in the Cath- $\mathrm{D}(++,+++)$ group was $47.6 \%(30 / 63)\left(\chi^{2}=6.623\right.$, $\mathrm{P}=0.036$ ) (Table I).

Relationship between hMAM mRNA expression in the BM and patient age. There was no correlation between hMAM expression and patient age $\left(\chi^{2}=1.056, \mathrm{P}=0.304\right)$ (Table I).

Relationship between hMAM mRNA expression in the BM and distant metastasis. Positive expression of hMAM mRNA in the BM before surgery was found in all 7 of the 8 patients who later developed distant metastases or who died. The quadruple tabular form exact probability test was used to examine the incidence of distant metastasis between the hMAM-positive and -negative groups $(\mathrm{P}=0.009)$.

\section{Discussion}

There are at least three conditions which must be fulfilled to define a perfect tumor marker. These are tumor specificity, tissue specificity and sensitivity. We found that a pseudo-gene was present when we detected the expression of CK-19 by RT-PCR (3), which made the micrometastasis rate higher than it really was. Therefore, it is difficult but rather important to choose a suitable marker.

hMAM is on chromosome 11q12-13, and the amino acid sequence of its translated protein is similar to that of a protein excreted by the epithelium, which belongs to the uteroglobulin family. They are all encoded by the genome in the same position on the chromosome. hMAM is expressed at lower levels in normal breast epithelium, but at higher levels in breast cancer tissue while hMAM is not expressed in normal blood and BM. Marchetti et al (4) researched micrometastasis to the lymph nodes in breast cancer patients and found that hMAM was more characteristic than the other 7 markers studied, such as CEA, CK-19 and MUC-1. The results of our study showed that the incidence rate of micrometastasis to the BM (hMAMpositive) was $38.2 \%$ in the group of 102 patients with primary breast cancer, while no micrometastasis was found in the group with benign breast diseases.

Since the 1980s, many animal experiments and clinical studies have found an intimate relationship between micrometastasis in the BM and poor prognosis of patients with breast cancer. Braun et al (5) carried out research on 552 patients with breast cancer, classified from grade 1 to grade 3 . He found that the rate of incidence of micrometastasis in the BM was 36.1\%. After a 4-year follow-up, 49 of 199 patients with micrometastasis to the BM died from cancer metastasis. In contrast, only 22 of 353 patients who were micrometastasisnegative died of cancer, which indicates that distant metastasis is positively correlated with the mortality rate of breast cancer patients who have micrometastasis to the BM.

The size of the tumor, the presence of axillary lymph node metastasis and clinical stage are all important with regard to the choice of therapy and prediction of prognosis. The common view is that prognosis gets worse in patients with larger tumors, metastasis to a greater number of axillary lymph nodes, or a more advanced clinical stage. Braun et al (6) carried out aggregate analysis of data from 4,199 breast cancer cases and obtained a rate of micrometastasis to the BM of $30.6 \%$. Substratificational analysis concluded that micrometastasis to the BM was correlated with the size of the tumor, the condition of axillary lymph node metastasis and histological classification $(\mathrm{P}<0.001)$. The 10 -year follow-up found that the total death rate in the BM micrometastasis-positive cases was higher than that of BM micrometastasis-negative group $(\mathrm{P}<0.001)$. The mortality rate was greatly increased in the BM micrometastasis-positive group, who also did not accept adjunctive therapy $(\mathrm{P}<0.001)$. The analysis indicated that the presence of micrometastasis in the BM was an independent predictor of prognosis. Furthermore, the impact on prognosis was much more obvious in the group in which the lymph nodes were metastasis-negative. Our results showed that the rate of micrometastasis to the BM of breast cancer patients increased as the tumor size increased or as the clinical stage increased. This indicates that the larger the tumor, the more invasive it is, which can promote the spreading of cancer cells to distant sites. However, nearly $10 \%$ of patients whose tumors were smaller than $2 \mathrm{~cm}$ or at stage I still expressed hMAM in their BM. At the same time, our results showed that the patients who were axillary lymph node metastasis-positive (52.9\%) had a higher expression of hMAM mRNA than the negative group (23.5\%), and this result was statistically significant $\left(\chi^{2}=14.050\right.$, $\mathrm{P}=0.001)$. This shows that there is a clear relationship between micrometastasis in the BM and axillary lymph node metastasis. We separated the positive group into two subgroups: A, those with 3 or fewer positive lymph nodes; and B, the remainder with 4 or more positive nodes. The results of ambi-comparisons showed that the rate of micrometastasis in the BM was higher in group $\mathrm{B}(\geq 4)$ than in the negative group and group $\mathrm{A}(\leq 3)$, a result which was statistically significant $\left(\chi^{2}=4.464,14.060\right.$ respectively; $\mathrm{P}=0.035,0.001$ respectively). That is to say the more metastasis-positive lymph nodes a patient has, the higher the rate of micrometastasis to the BM. However, there was no obvious difference between the negative group and group A, and the difference was not statistically significant $\left(\chi^{2}=1.881\right.$, $\mathrm{P}=0.170)$. Previous clinical studies have found that approximately $20-30 \%$ of patients with no metastasis in the lymph nodes suffer from metastasis and recurrence, which leads to death within the first 5 years after surgery. Our study also found that various patients with no metastasis to the lymph nodes, and several of those with fewer than 3 positive lymph nodes, also expressed hMAM mRNA in their BM; the rate of expression being 23.5 and $61.5 \%$, respectively. These findings show that breast cancer may be a systemic disease, and that 
hematogenous metastasis can occur even during the initial stage. Our team also reached this conclusion by detecting micrometastasis in the BM by means of CK-19 (7). Clinical studies have found that certain patients with small tumors and at initial stages die soon after surgery as a result of metastasis; the reason may have some relationship with this finding. These observations emphasize the need for these patients to receive systemic adjunctive therapy to improve their long-term survival rate. We also consider that the detection of axillary lymph node metastasis is insufficient to judge prognosis and to direct general treatments, especially when the patients have no metastasis in the axillary lymph nodes or have fewer positive lymph nodes. Therefore, it is necessary to detect micrometastasis in the BM during routine examination. Assessment of the level of metastasis throughout the body much earlier and more precisely is critical. As a result, we can then offer patients with micrometastasis active systemic therapy, and avoid overtreatment, even in cases in which the clinical stage is more favorable, tumor size is smaller or the axillary lymph nodes are metastasis-negative.

Micrometastasis in the BM of breast cancer patients has a certain relationship with histological classification. The rate of hMAM mRNA expression in the BM of patients was higher in poorly differentiated cases than in those with good differentiation. The rate of micrometastasis in BM was higher in the group with infiltrating ductal carcinoma and infiltrating lobular carcinoma than the group with types of breast cancer with lower malignant potential. Thus, the types of breast cancer with poor differentiation and higher potential of malignancy have much greater abilities to proliferate, invade and migrate.

Stathopoulou et al (9) and Ooka et al (10) found that detection of micrometastasis was correlated with diseasefree and total survival, providing a reference with which to judge prognosis. Our results showed that the 7 patients who later suffered distant metastasis and death were all hMAM mRNA-positive before surgery. Statistical analysis indicated a strong correlation between the detection of hMAM and metastasis in patients.

The present study found that the BM micrometastasis rate was high in the group that were ER, PR negative expression and the group that were ER, PR low expression, however the BM micrometastasis rate declined as the expression of ER and PR increased, $\mathrm{P}<0.05$. According to Braun et al (6) the BM micrometastasis rate was high in the ER negative expression group. This indicated that the hematogeneous metastasis risk would increase in cases which were ER, PR negative expression or ER, PR low expression, and that cells identified as ER, PR negative expression, possibly, had greater invasive ability. Breast cancer cases identified as ER, PR low expression had a poor prognosis in comparison with ER, PR positive expression cases.

Cath-D is a type of lysosomal enzyme; its physiologic function is to degrade protein in the acid environment of the lysosomes. By accelerating tumor cell growth, decreasing the activity of tumor-inhibiting factor (TIF), and degrading basal lamina, Cath-D increases the propensity of the tumor cells to invade and metastasize. Wu et al (11) found that Cath-D hyperexpression indicated poor prognosis. Our study showed that the $\mathrm{BM}$ micrometastasis rate rose as the expression of Cath-D increased $(\mathrm{P}<0.05)$.

In conclusion, the detection of micrometastasis in the $\mathrm{BM}$ can be one way to assess the prognosis of breast cancer. Using dynamic detection, we can quickly obtain information concerning therapeutic efficacy, monitor changes in the patient's condition, adjust the therapeutic regimen and evaluate progress. This molecular prognostic method can play an active part in clinical research, and it can provide a basis for an individual therapeutic regimen for each patient with breast cancer.

\section{Acknowledgements}

This study has supported by the Key Oncologic Subject Foundation of Hebei Province (no. 200552), the Natural Science Foundation of Hebei Province.

\section{References}

1. Carcoforo P, Bergossi L, Basaglia E, et al: Prognostic and therapeutic impact of sentinel node micrometastasis in patients with invasive breast cancer. Tumori 88: S4-S5, 2002.

2. Gebauer G, Fehm T, Merkle E, Beck EP, Lang N, and Jäger W: Epithelial cells in bone marrow of breast cancer patients at time of primary surgery: clinical outcome during long-time follow-up. J Clin Oncol 19: 3669-3674, 2001.

3. Feng YM, Hao XS, Yu Y, Wang LM and Li X: The pseudogene interference of Ck-19 as marker to detect the gene of micrometastasis carcinoma and the means to solve the interference. Chin J Oncol 23: 330-331, 2001 (In Chinese).

4. Marchetti A, Buttitta F, Bertacca G, et al: mRNA markers of breast cancer nodal metastases: comparision between mammaglobin and carcinoembryonic antigen in 248 patients. J Pathol 195: 186-190, 2001.

5. Braun S, Pantel K, Muller P, et al: Cytokeratin-positive cells in the bone marrow and survival of patients with stage I, II, or III breast cancer. N Engl J Med 342: 525-533, 2000.

6. Braun S, Vogl FD, Naume B, et al: A pooled analysis of bone marrow micrometastasis in breast cancer. N Engl J Med 353: 793-802, 2005.

7. Liu YJ, Wu FY and Wu XD: Detection of micrometastasis in bone marrow of patients with breast cancer and its clinical significance. J Clin Surg 12: 527-529, 2004 (In Chinese).

8. Fabisiewicz A, Kulik J, Kober P, Brewczyńska E, Pieńkowski T, and Siedlecki JA: Detection of circulating breast cancer cells in peripheral blood by a two-marker RT-PCR assay. Acta Biochim Pol 51: 747-755, 2004.

9. Stathopoulou A, Vlachonikolis I, Mavroudis D, et al: Molecular detection of cytokeratin-19-positive cells in the peripheral blood of patients with operable breast cancer: evaluation of their prognostic significance. J Clin Oncol 20: 3404-3412, 2002.

10. Ooka M, Tamaki Y, Sakita I, et al: Bone marrow micrometastases detected by RT-PCR for mammaglobin can be an alternative prognostic factor of breast cancer. Breast Cancer Res Treat 67: 169-175, 2001.

11. Wu FY, Liu YJ, Wang XL, Yang HC and Wang YJ: The expression of cathepsin-D in breast cancer tissue and the correlation with other related targets. J Tumor Treat Prev 12: 190-192, 2005 (In Chinese). 